\title{
Startup of anaerobic biofilm fluidized bed reactors on molasses and phenol under various conditions
}

\author{
I. J. Dunn and S. Petrozzi, Zurich
}

\begin{abstract}
Fluidized sand bed anaerobic biofilm reactors were operated in parallel to study the effects of inoculum, loading, residence time and carrier type on the startup dynamics for the degradation of molasses and phenol. Degradation rates generally depended most directly on concentrations rather than on other operating variables. Residence times did not appear to directly influence startup. Short residence times and high loadings gave the highest specific activities for both substrates. The type of inoculum was found to be most important for the molasses system, and inoculation on fresh carrier was found to be better than reinoculation. The two times higher specific biomass retention on Siran porous glass gave essentially the same degradation rates on a volume basis.
\end{abstract}

\section{List of symbols}

$L \quad \mathrm{~kg} / \mathrm{h}$

$M \quad \mathrm{~kg} / \mathrm{kg}$

Red. $\%$

$R \quad \mathrm{~kg} /\left(\mathrm{m}^{3} \cdot \mathrm{h}\right)$

$S \quad \mathrm{~kg} / \mathrm{m}^{3}$

$S_{0} \quad \mathrm{~kg} / \mathrm{m}^{3}$

$t \quad \mathrm{~b}$ inoculum from fluidized beds [5]. One study used different inocula from anaerobic fluidized beds and short residence times, and concluded that the reactor conditions are more important than the inoculum [6]. Few studies have performed parallel experiments with a multireactor system to test the above important operating variables.

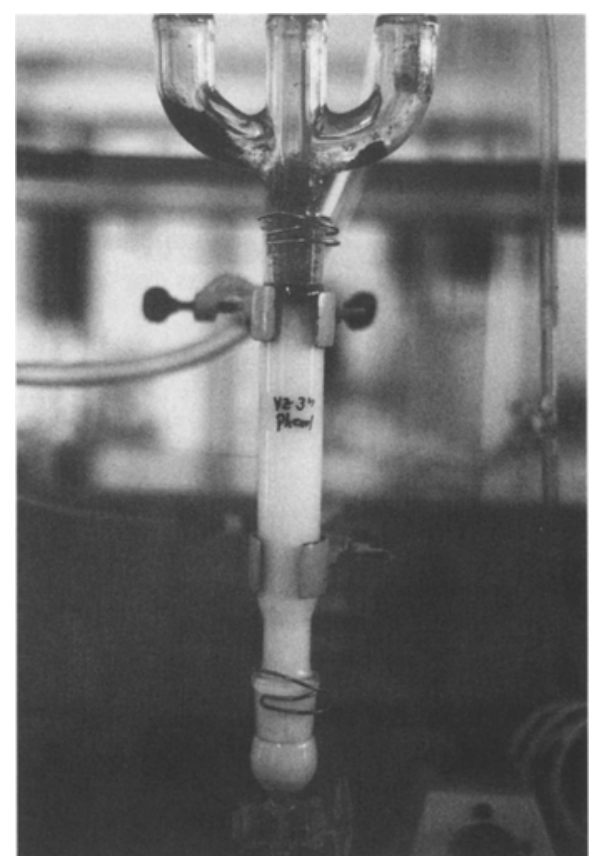

Fig. 1. Photo of the anaerobic fluidized bed reactor

Table 1. Reactor conditions

\begin{tabular}{ll}
\hline Temperature: & $37^{\circ} \mathrm{C}$ \\
pH: & $6.5-7$ \\
Reactor volume: & $425 \mathrm{~cm}^{3}$ \\
Fluidization velocity: & $20 \mathrm{~m} / \mathrm{h}$ \\
Residence times: & 3,36, and $108 \mathrm{~h}$ \\
Carriers: & Sand $(0.3 \mathrm{~mm})$ and porous glass (Siran) \\
Carrier quantity: & $70 \mathrm{~cm}^{3}$ (settled volume) \\
Bed expansion: & $150-200 \%$ \\
Substrates: & molasses and phenol \\
\hline
\end{tabular}


In the present work the objective was to test with parallel reactors the variables of residence times and loading for an easily degradable substrate and a more difficult inhibitory substrate. In addition, the comparison of a porous glass carrier with rough sand was of interest.

\section{Methods}

The reactors consisted of standard glass parts fitted together with tapered joints. As seen in the photo of Fig. 1 the column $\left(3.3 \mathrm{~cm}\right.$ diameter and $33 \mathrm{~cm}$ height, $450 \mathrm{~cm}^{3}$ volume) was topped with a three armed settling section. Circulation and fluidization flow rates were obtained with peristaltic pumps. The carrier was crushed quartz sand $(0.25-0.35 \mathrm{~mm})$ in the case of molasses feed, and in the case of phenol feed both sand and Siran (Schott Co.) porous glass $(0.4-0.6 \mathrm{~mm}$ diameter, 0.55 porosity) were used. The same settled carrier volume of $70 \mathrm{~cm}^{3}$ was used in each reactor.

With molasses feed, $\mathrm{pH}$ control was made with $0.2 \mathrm{~N}$ $\mathrm{NaOH}$ using an electrode with gel electrolyte (Ingold), especially suited for anaerobic systems. The reactors were thermostated with a heat exchanger in the circulation line. In Table 1 are summarized the operating conditions of the reactors $(R 1-R 10)$. In Table 2 are given the conditions of the individual reactor startup experiments.

Analysis of molasses and phenol were made using a commercial colorimeter test system (Nanocolor) using the $C O D$ and phenol test kits. Biomass was obtained as volatile carbon by analyses with a TOC instrument (Dohrmann). Gas production was measured by a volumetric gas meter and composition was determined by GC.

The feed consisted of either molasses $(1 \mathrm{~g}$ molasses $=$ $0.72 \mathrm{~g} C O D$ ) or phenol in tap water. In addition nutrients salts were added (in $\mathrm{kg} / \mathrm{m}^{3}$ ): $0.13 \mathrm{CaCl}_{2}, 0.5 \mathrm{Mg} \mathrm{Cl}$, $0.2 \mathrm{NH}_{4} \mathrm{Cl}$. Trace element solution $\left(1 \mathrm{~cm}^{3} / \mathrm{dm}^{3}\right)$ containing $\mathrm{Fe}, \mathrm{Mn}, \mathrm{Zn}, \mathrm{Co}, \mathrm{Ni}, \mathrm{B}, \mathrm{Mo}, \mathrm{Co}, \mathrm{V}$, citrate and cystine was also added.

The startup experiments were run in parallel with five reactors. Two different types of inocula were used, a mixture of industrial activated sludges (AS) and a very active anaerobic biofilm (ABF) from a whey culture. The feed was either molasses or phenol.

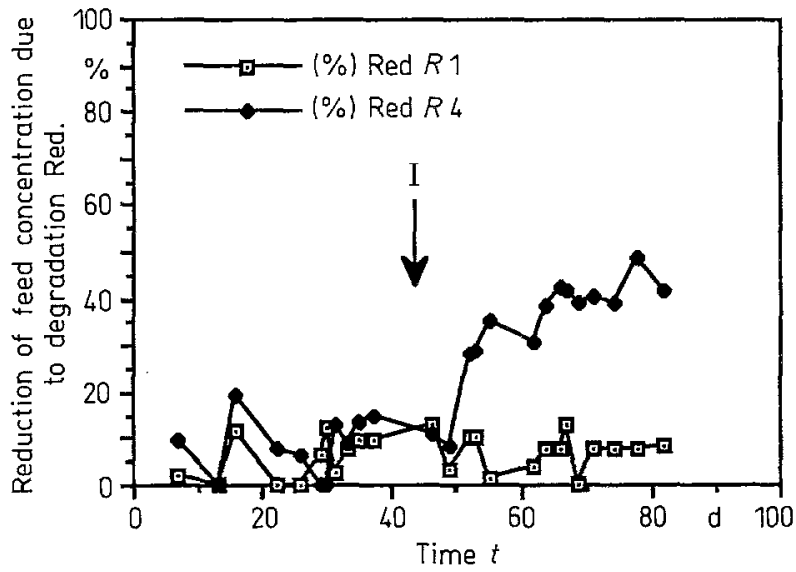

Fig. 2. Comparison of the startup of two reactors on molasses operating at two different residence times, $R 1-3 \mathrm{~h}$ and $R 4-36 \mathrm{~h}$. Reinoculation with very active anaerobic biofilm at day 45 . Percent reduction is plotted versus time

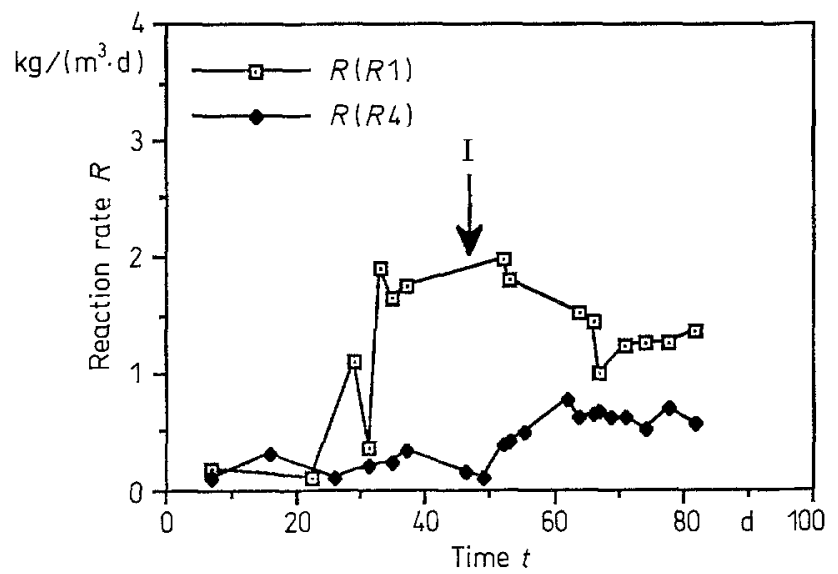

Fig. 3. Comparison of the startup of two reactors on molasses operating at two different residence times, $R 1-3 \mathrm{~h}$ and $R 4-36 \mathrm{~h}$. Degradation rate is plotted versus time

\section{Discussion of results}

\subsection{Startup on molasses with reinoculation ( $R 1$ and $R 4$ )}

The results from the 45 day startup of the sand beds inoculated with activated sludge ( $R 1$ and $R 4$ ) are given Figs. 2-4.

Table 2. Reactor conditions

\begin{tabular}{|c|c|c|c|c|c|c|}
\hline Carrier & Reactor & $\begin{array}{l}\text { Residence time } \\
{[\mathrm{h}]}\end{array}$ & Feed conc. & $\begin{array}{l}\text { Load } \\
{\left[\mathrm{kg} / \mathrm{m}^{3}\right]}\end{array}$ & $\begin{array}{l}\text { Substrate } \\
{\left[\mathrm{kg} /\left(\mathrm{m}^{3} \cdot \mathrm{d}\right)\right]}\end{array}$ & Inoculum \\
\hline Sand & $R 1$ & 3 & 3 & 24 & Molasses & $\mathrm{AS}+\mathrm{ABF}$ \\
\hline Sand & $R 7$ & 3 & 3 & 24 & Molasses & $\mathrm{ABF}$ \\
\hline Sand & $R 8$ & 36 & 3 & 2 & Molasses & $\mathrm{ABF}$ \\
\hline Sand & $R 9$ & 108 & 3 & 0.67 & Molasses & $\mathrm{ABF}$ \\
\hline Sand & R10 & $108-112$ & $3-12$ & $2-24$ & Molasses & $\mathrm{ABF}$ \\
\hline Sand & $R 5$ & 36 & $0.02-0.25$ & $0.01-0.17$ & Phenol & $\mathrm{AS}+\mathrm{ABF}$ \\
\hline
\end{tabular}




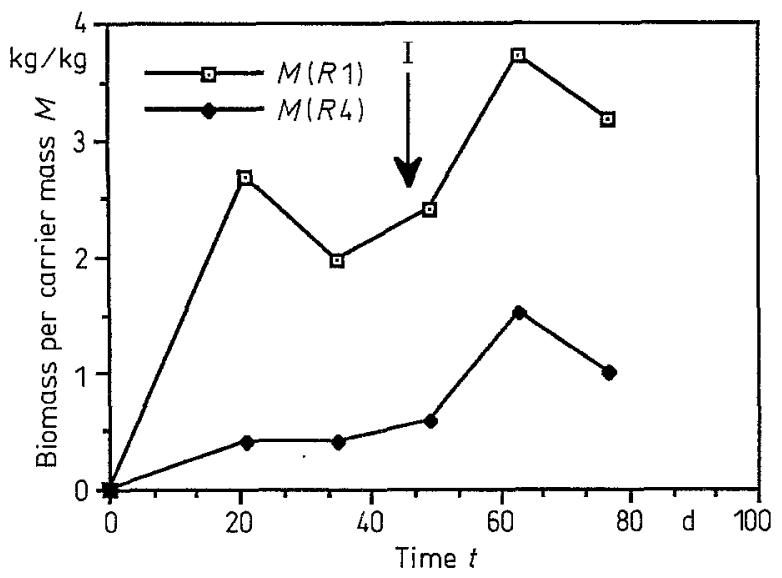

Fig. 4. Comparison of the startup of two reactors on molasses operating at two different residence times, $R 1-3 \mathrm{~h}$ and $R 4-36 \mathrm{~h}$. Biomass on carrier is plotted versus time

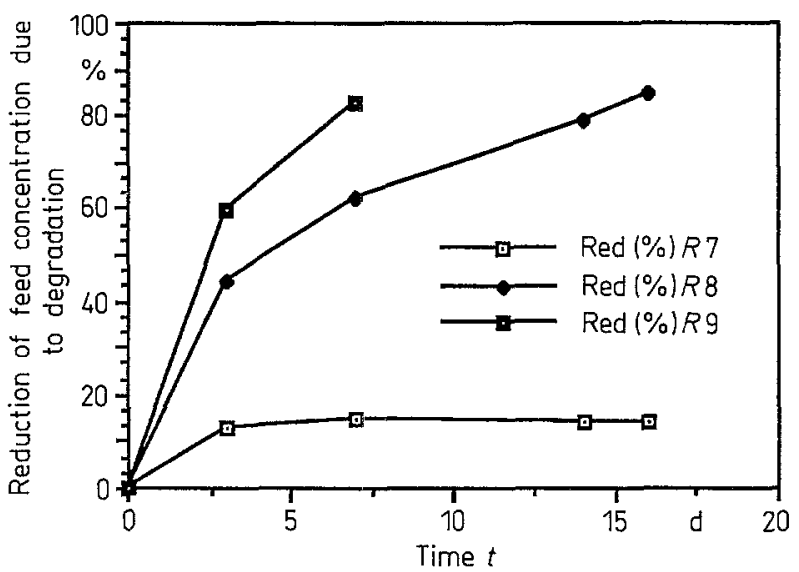

Fig. 5. Startup of three reactors on molasses with biofilm inoculum operating at three different residence times, $R 7-3 \mathrm{~h}, R 8-36 \mathrm{~h}$, and $R 9-108 \mathrm{~h}$. Percent reduction is plotted versus time

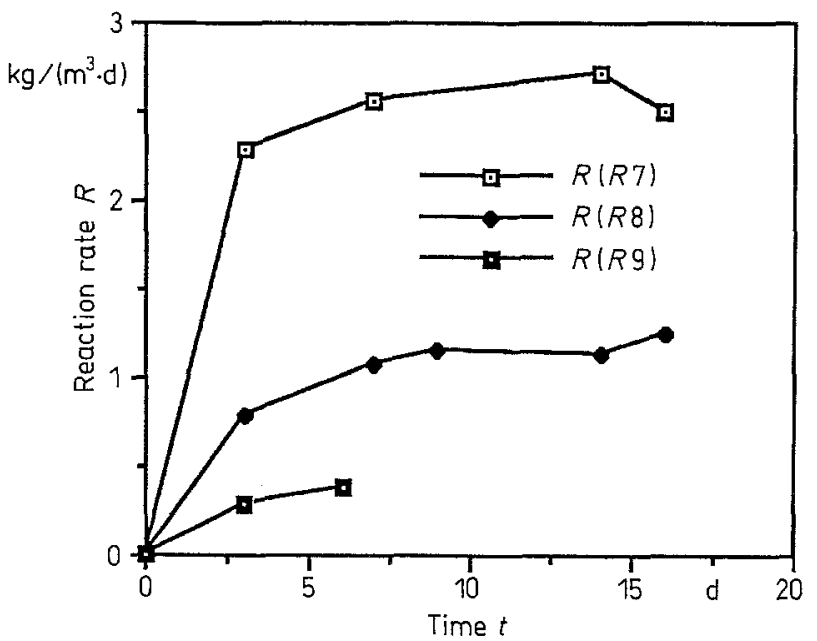

Fig. 6. Startup of three reactors on molasses with biofilm inoculum operating at three different residence times, $R 7-3 \mathrm{~h}, R 8-36 \mathrm{~h}$, and $R 9-108 \mathrm{~h}$. Degradation rate is plotted versus time

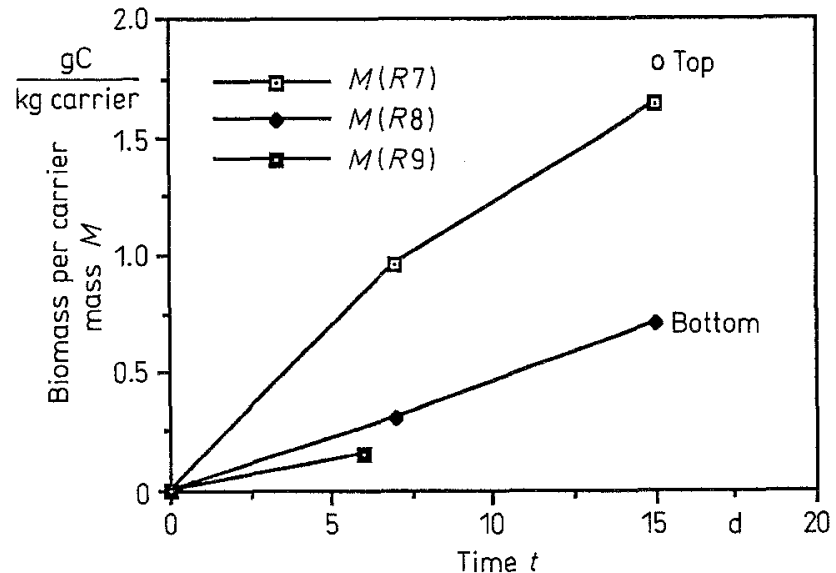

Fig. 7. Startup of three reactors on molasses with biofilm inoculum operating at three different residence times, $R 7-3 \mathrm{~h}, R 8-36 \mathrm{~h}$, and $R 9-108 \mathrm{~h}$. Biomass on carrier is plotted versus time

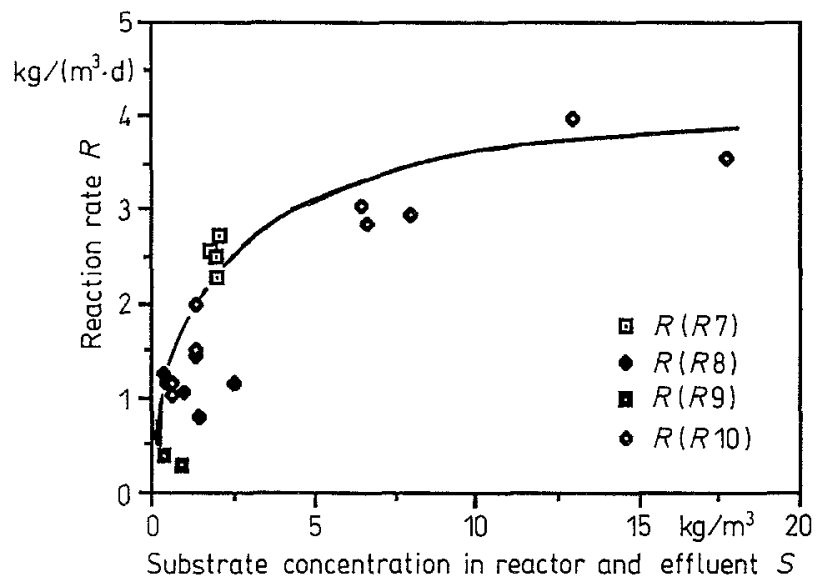

Fig. 8. Rate versus concentration for the startup of three reactors on molasses with biofilm inoculum operating at three different residence times, $R 7-3 \mathrm{~h}, R 8-36 \mathrm{~h}$, and $R 9-108 \mathrm{~h}$

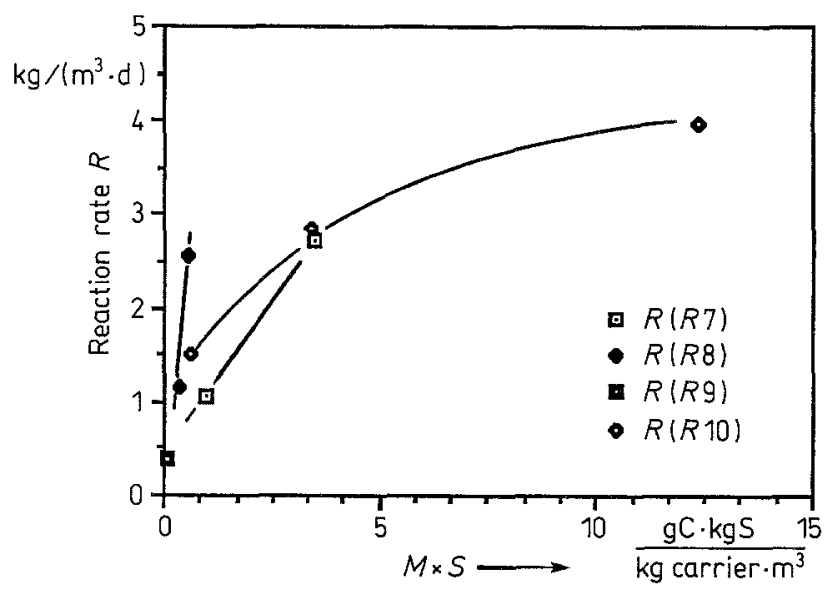

Fig. 9. Rate versus concentration times biomass for the startup of three reactors on molasses with biofilm inoculum operating at three different residence times, $R 7-3 \mathrm{~h}, R 8-36 \mathrm{~h}$, and $R 9-108 \mathrm{~h}$ 
The reactors ran for 6 weeks with a thick biofilm development, but without gas production. At day 45 the reactors were reinoculated with anaerobic biofilm organisms. During the first period the COD reduction remained below $20 \%$ (Fig. 1 a). After reinoculation the reactor with $36 \mathrm{~h}$ residence time (R4) reached $45 \%$ COD reduction. The reactor with $3 \mathrm{~h}$ residence time $(R 1)$ was not appreciably influenced by the inoculum. This difference in reactor performance is seen best in Fig. 3, in which the degradation rates are plotted. $R 1$ actually exhibited decreased rates after inoculation.

TOC analysis of the biomass on the sand carrier gave the data of Fig. 4: reactor $R 1$ had the most biofilm development in agreement with its highest rates. Both $R t$ and $R 4$ increased their biofilm after reinoculation. It is not known why this increase did not give a rate increase in the case of R1.

\subsection{Startup on molasses with biofilm inoculation (R7, R8, R9 and R10)}

Three sandbed reactors were inoculated with the anaerobic biofilm organisms and fed with molasses $\left(3 \mathrm{~kg} / \mathrm{m}^{3}\right)$ at residence times of $3 \mathrm{~h}(R 7), 36 \mathrm{~h}(R 8)$, and $108 \mathrm{~h}(R 9)$. After a few days methane was produced and high degradation rates were obtained. The loads, as seen in Table 2, were 24.0, 2.0 and $0.67 \mathrm{~kg} / \mathrm{m}^{3}$ day. In Figs. 5-7 the data from these startup experiments are shown. The lower the loading was the higher were the percent reductions. The reaction rates were highest for the highest loadings and followed Monod kinetics, as shown by the plot of Fig. 8 in which the variation of rate with reactor concentration for all the reactors is given.

The variation of biomass (as biofilm) with time, from samples taken near the center of the column, is given in Fig. 7. The lowest residence times and highest loading gave the highest biomass concentrations. The single data point from top of $R 8$ at day 15 demonstrates that stratification causes the biomass to concentrate at the top of the column.

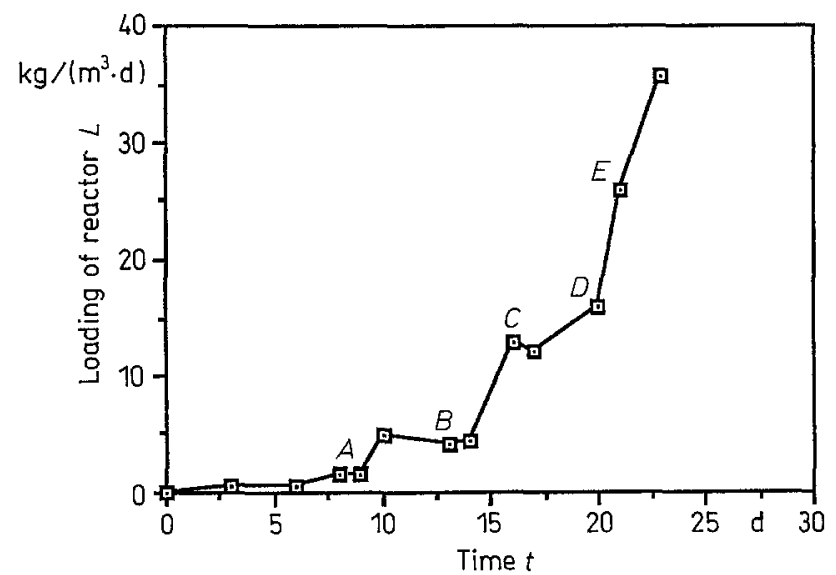

Fig. 10. Loading of $R 10$ versus time. The periods are designated $\mathrm{A}$, $\mathrm{B}, \mathrm{C}$, and D

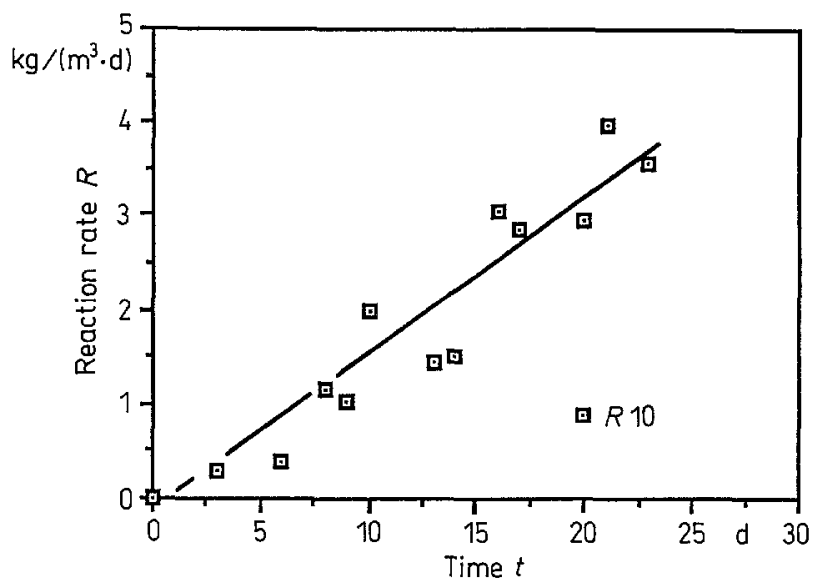

Fig. 11. Rate versus time for $R 10$

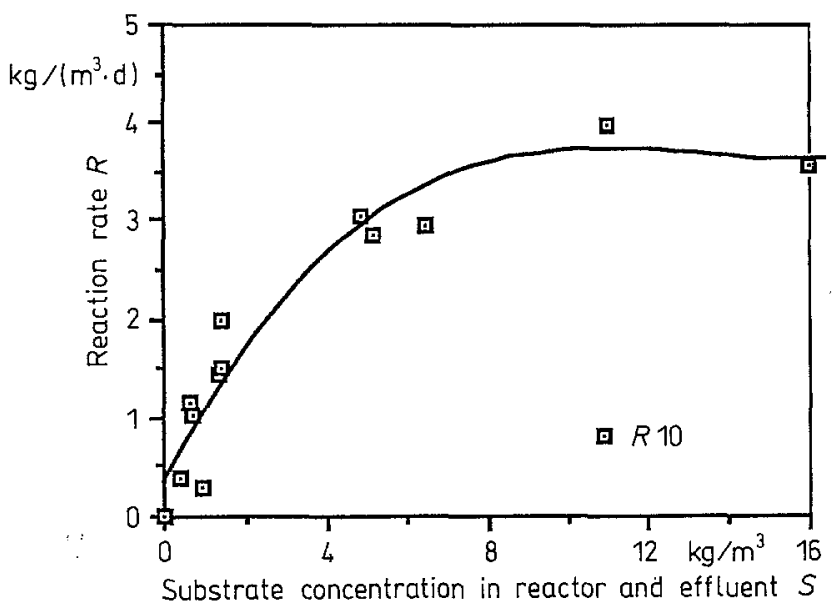

Fig. 12. Rate versus concentration for $R 10$

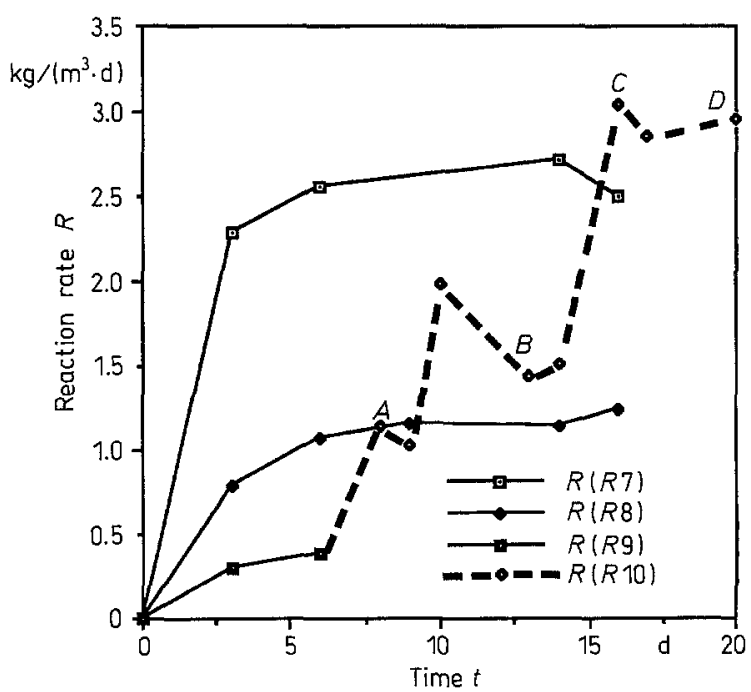

Fig. 13. Rate versus time for $R 10$ showing $R 7$ and $R 8$ as comparison 


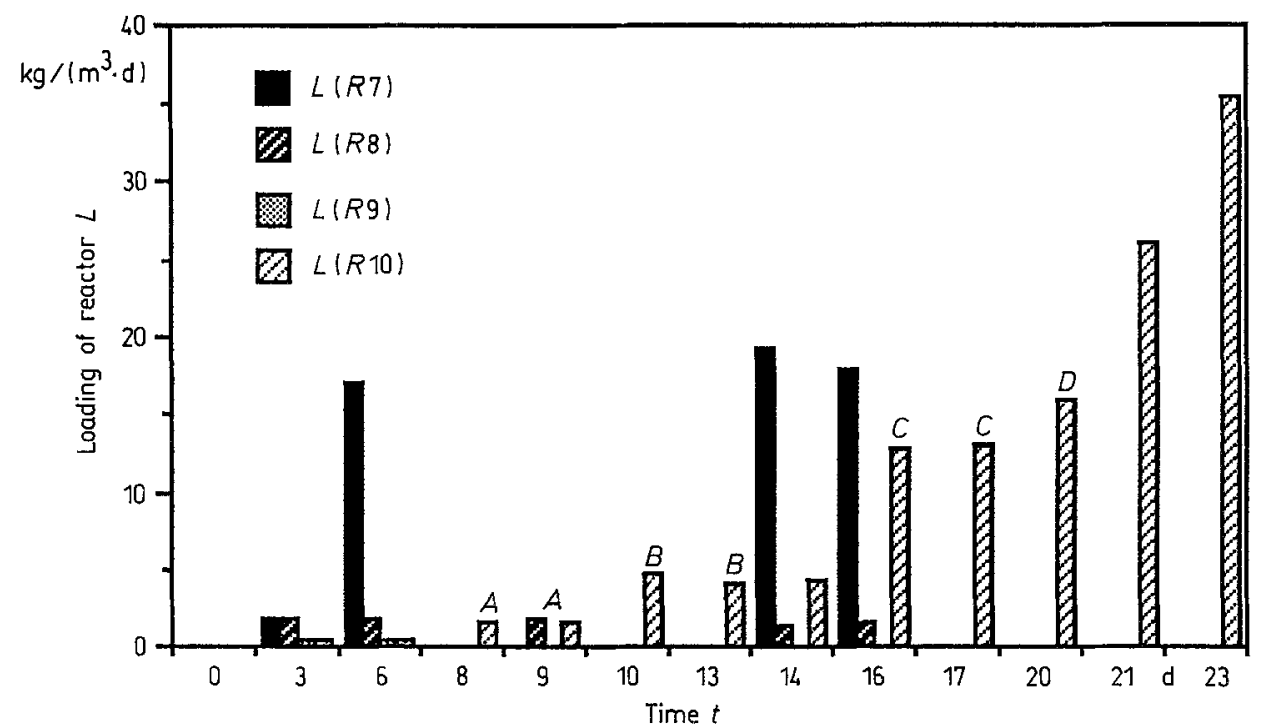

Fig. 14. Loading of $R 10$ showing $R 7$ and $R 8$ as comparison

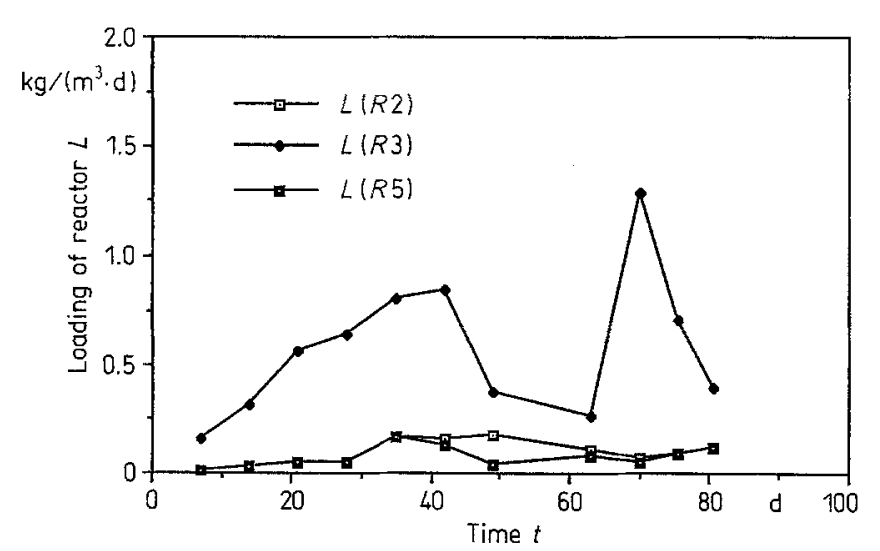

Fig. 15. Loading of $R 2, R 2$ and $R 5$ with phenol during an 80 day startup period

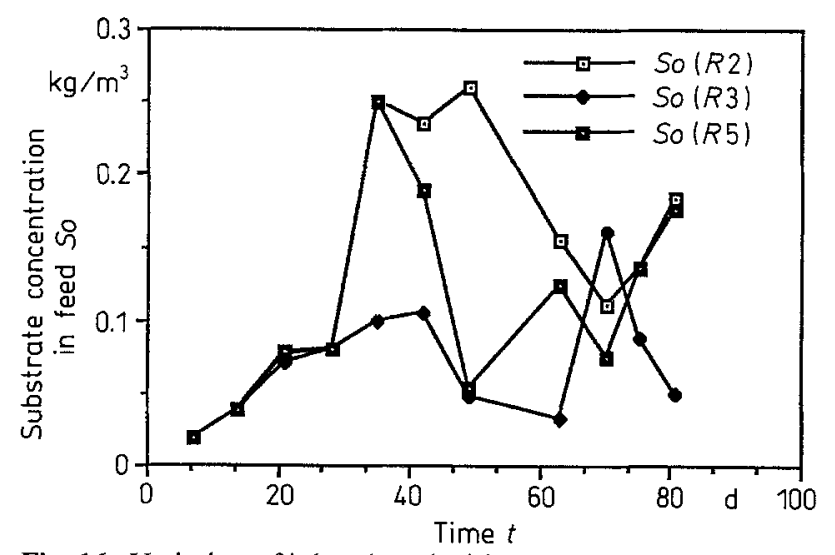

Fig. 16. Variation of inlet phenol with $R 2, R 3$ and $R 5$ during the 80 day startup period

It is not known why the biomass data of Fig. 7 are generally lower than those of Fig. 4 , even though the rates for $R 7, R 8$, and $R 9$ were higher than those for $R 1$ and $R 4$. The explanation for this is probably the lack of methanogens in $R 1$ and $R 4$. Again, the rates follow a Monod function shape with concentration, as seen in Fig. 8. Plotting the rate data versus
$M \times S$ for the limited data available, gave the results in Fig. 9. Data for $R 9$ with increased loading, designated $R 10$, to be explained later, are also included in these figures. One might have expected the points to fall along one line, signifying equal rate constants. The sensitivity of the rate in $R 7$ to biomass may be due to a higher specific biomass activity, which would tend to be promoted by the lower residence time.

The loading of $R 9$ was increased after day 6 by changing the residence times and feed concentrations in sequential steps as follows: Period A: $t=36 \mathrm{~h}, S_{0}=3$; Period B: $t=12$, $S_{0}=3$; Period C: $t=9, S_{0}=12$; Period D: $t=12, S_{0}=12$. As seen in Fig. 10, the loading thus changed from 0.66 in $R 9$ to 2.0 , to 6.0 , to 18.0 and to $24.0 \mathrm{~kg} / \mathrm{m}^{3}$ - day). In Fig. 11 the increased loads are seen to have caused increases in degradation rate, which followed a Monod function with concentration (Fig. 12). As a comparison, the rate data from $R 7$ and $R 8$ are shown in Fig. 13. The constant operating conditions of $R 8$ were exactly the same as those of $R 10$ in period $\mathrm{A}$, while period $\mathrm{D}$ corresponded to the same loading as $R 7$. Noteworthy is that $R 10$ responded quickly to the load changes and was able to reach the same rates within a short time as were attained by $R 7$ and $R 8$ during long-term constant conditions. The loadings of $R 7, R 8, R 9$ and $R 10$ are compared in Fig. 14. The loads of $R 7, R 8$ and $R 9$ were maintained essentially constant, while $R 10$ was increased to load levels equivalent to $R 8$ during days $8-10$ and finally to levels in the range of $R 7$ during days 16-22.

\subsection{Startup on phenol with sand and porous glass carriers}

As summarized in Table 2, reactors $R 2, R 3$ and $R 5$ were run with phenol as substrate. The carrier in $R 2$ was Siran porous glass, and in $R 3$ and $R 5$ sand was used. The residence times were $36 \mathrm{~h}$ for $R 2$ and $R 5$ and $3 \mathrm{~h}$ for $R 3$. The feed concentrations were increased to maintain adequate levels in the reactor (Figs. 15 and 16). The resulting effluent (and reactor concentrations) are given in Fig. 17. The reaction rates and 


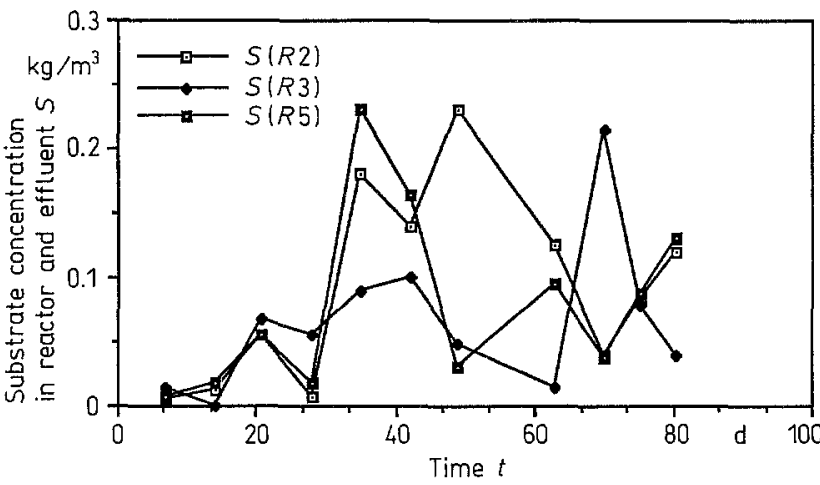

Fig. 17. Variation of effluent phenol with $R 2, R 3$ and $R 5$ during the 80 day startup period

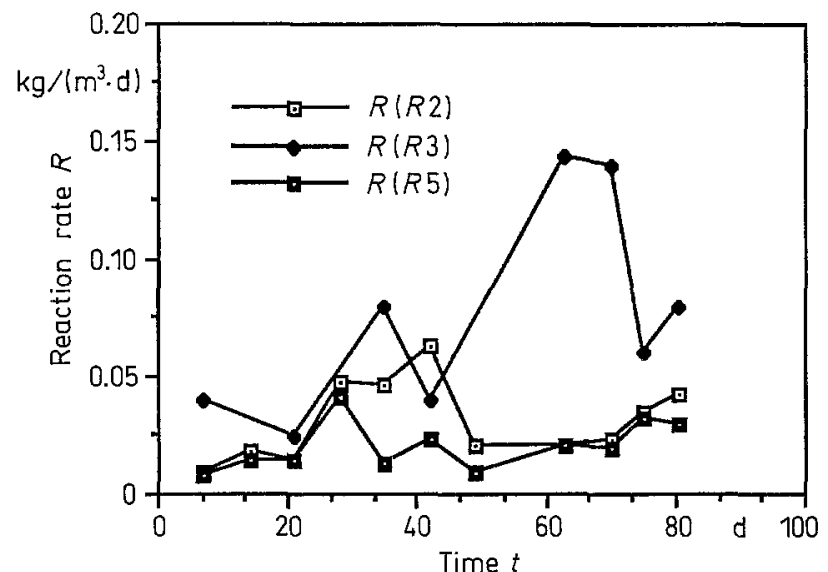

Fig. 18. Rate versus time for $R 2, R 3$ and $R 5$ during the 80 day startup period

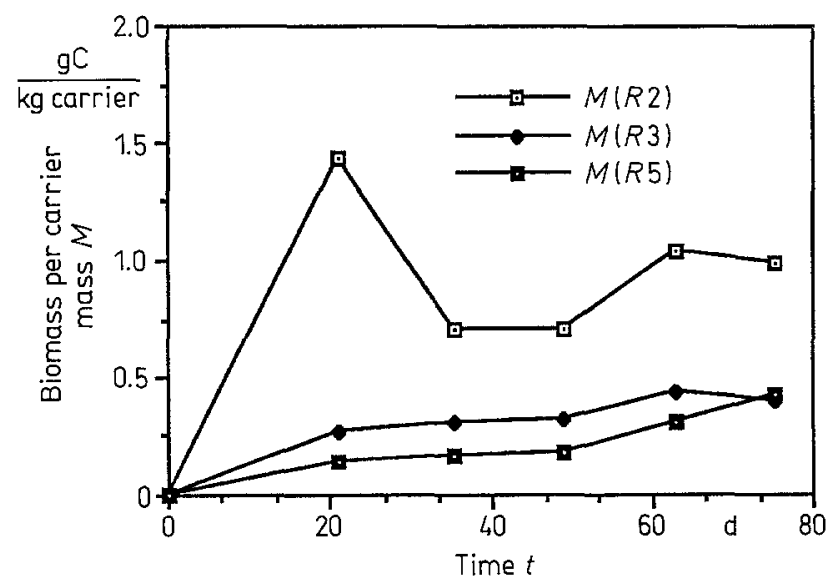

Fig. 19. Biomass on carrier versus time for $R 2, R 3$ and $R 5$ during the 80 day startup period

biomass levels over the 80 day period are given in Figs. 18 and 19. It is interesting that $R 2$ (porous glass) had the highest biomass level, but had a reaction rate comparable to $R 5(t=36 \mathrm{~h}) . R 3$ (sand $t=3 \mathrm{~h}$ ) exhibited substantially higher rates. Plotted versus phenol concentration the rates are seen to increase with concentration for all reactors (Fig. 20). $R 2$

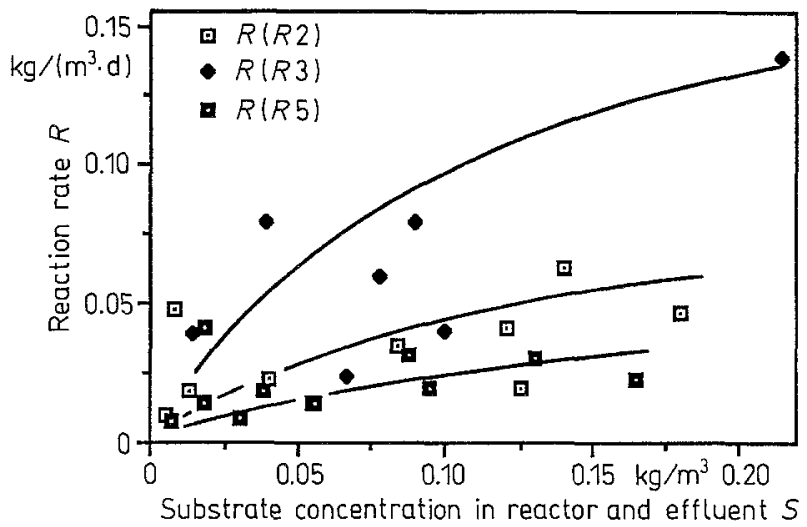

Fig. 20. Rate versus concentration for $R 2, R 3$ and $R 5$ during the 80 day startup period

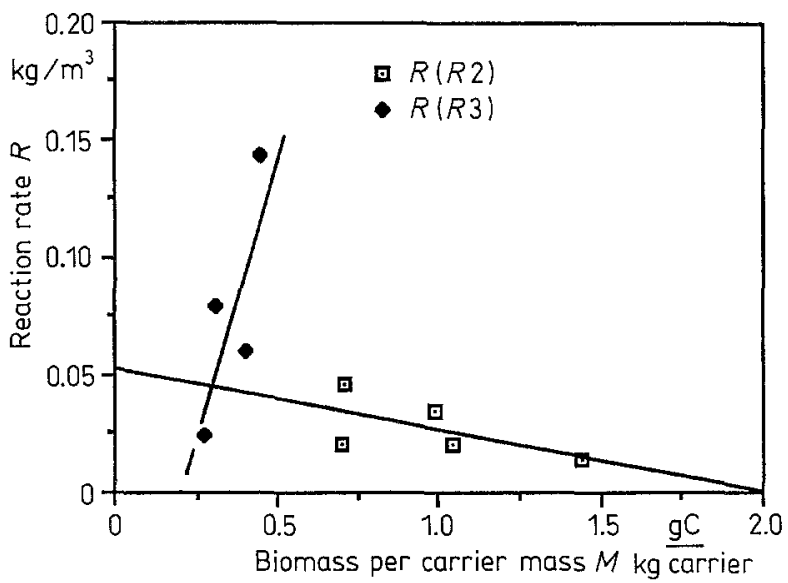

Fig. 21. Rate versus biomass for $R 2$ and $R 3$ during the 80 day startup period

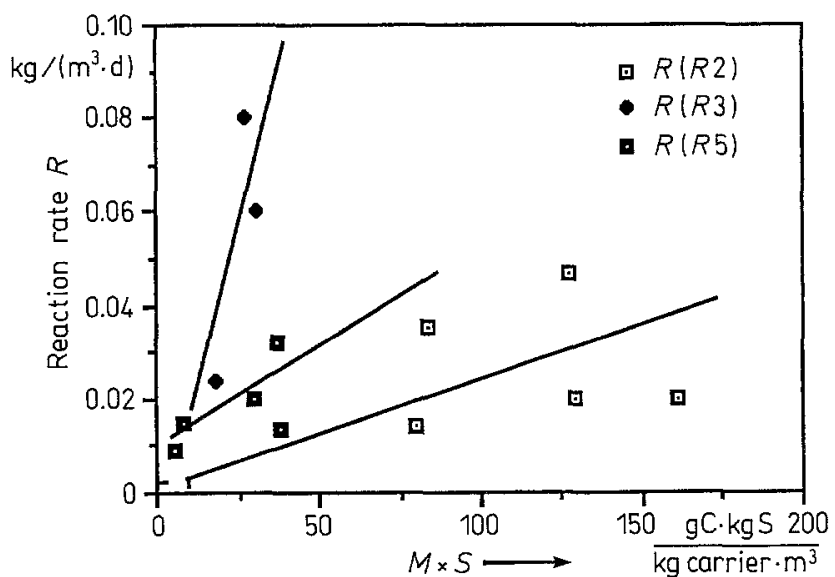

Fig. 22. Rate versus concentration times biomass for $R 2, R 3$ and $R 5$ during the 80 day startup period

exhibited a decrease of rate with biomass (Fig. 21), whereas $R 2$ increased sharply with biomass. Taking both biomass and concentration into account (by plotting rate versus $M \times S$ in Fig. 22) shows that the data from $R 2$ and $R 5$ exhibit comparable slopes, but the data from $R 3$ have a much higher slope. Similar to the molasses reactor, the low 


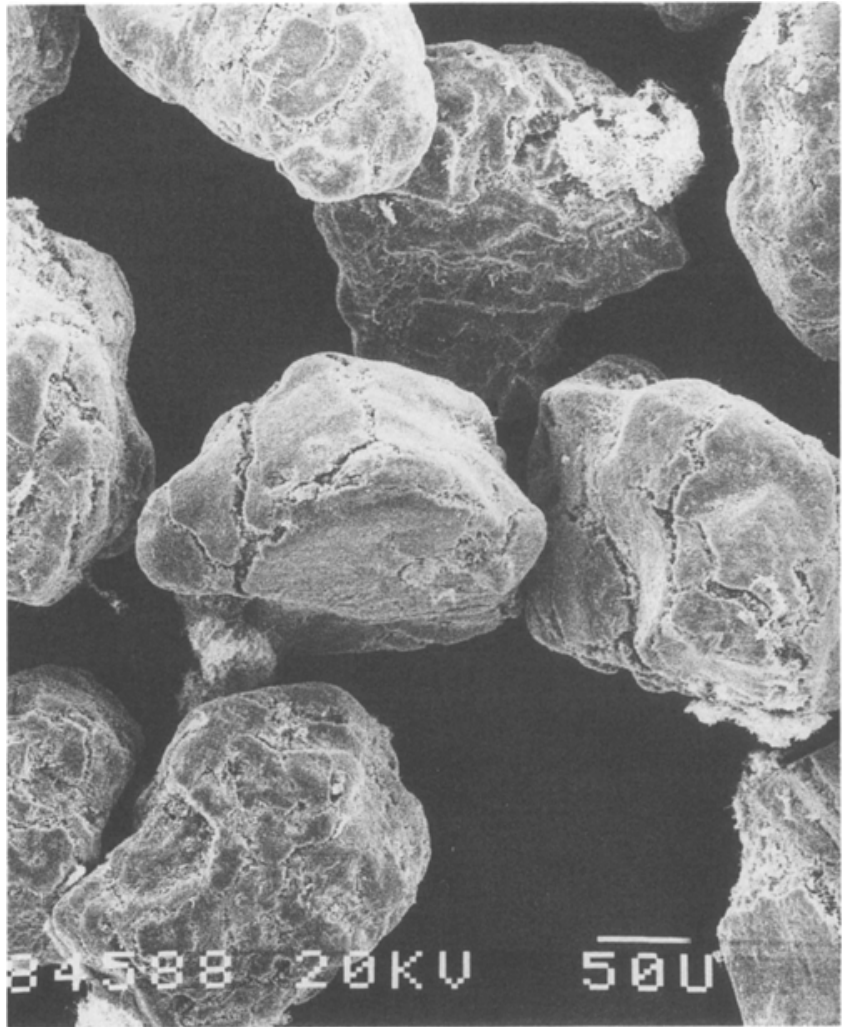

Fig. 23

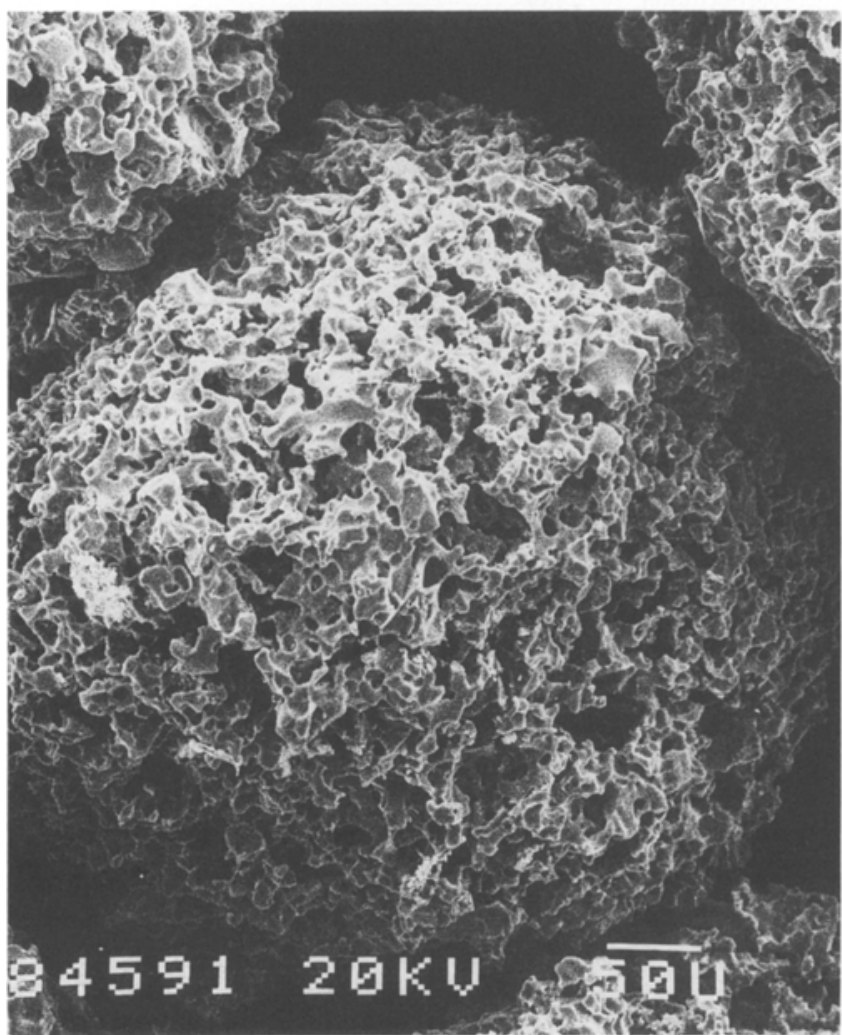

Fig. 25

Fig. 23. Photo of molasses degrading anaerobic biofilm on sand particles (approximately $200 \mu \mathrm{m}$ diameter)

Fig. 24. The same biofilm as in Fig. 23, at higher magnification

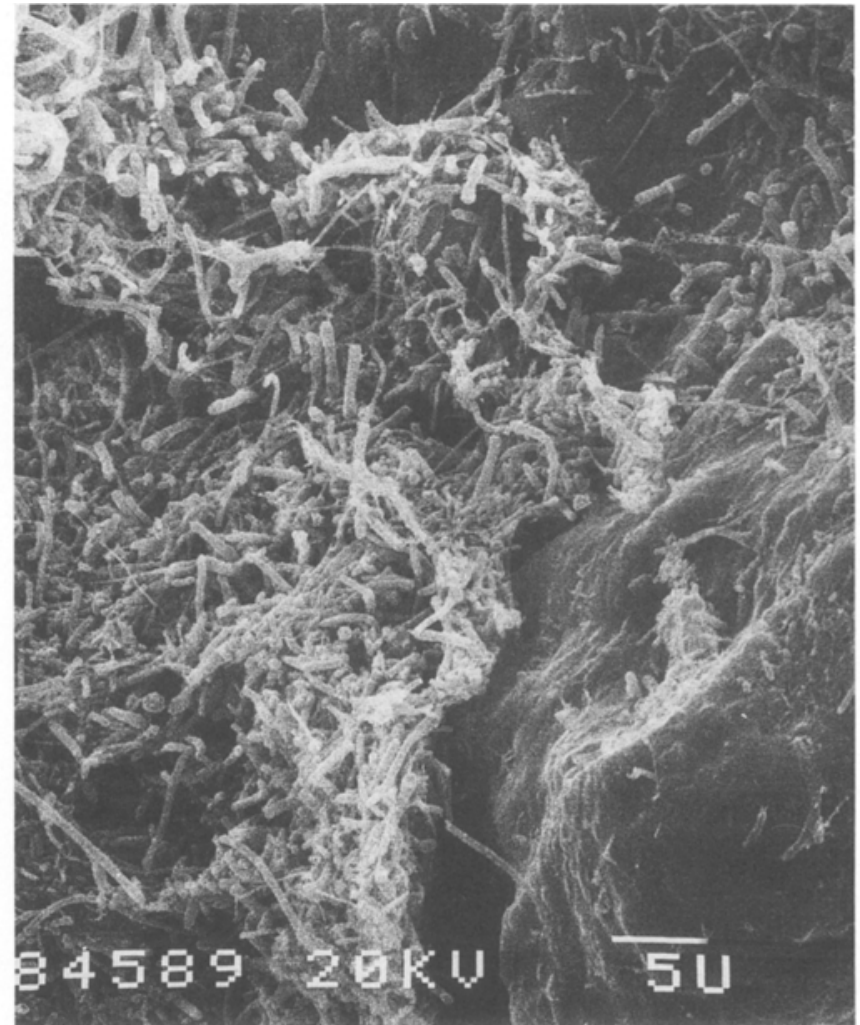

Fig. 24

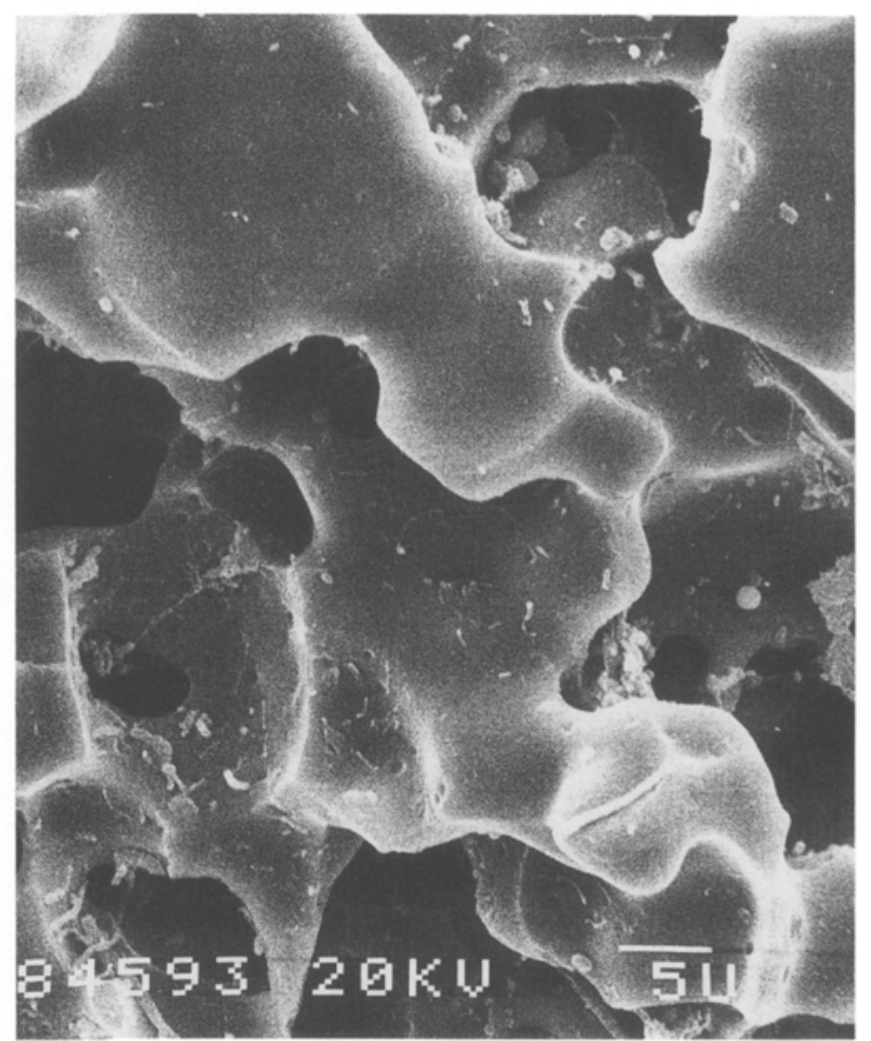

Fig. 26

Fig. 25. Photo of phenol degrading anaerobic biofilm on porous glass particles (approximately $200 \mu \mathrm{m}$ diameter)

Fig. 26. The same carrier and biofilm as in Fig. 25, at the same magnification as in Fig. 24 
residence time condition appeared to have higher specific biomass activity. It should be noted that the rates are low and, especially for $R 3$, if the differences between inlet and outlet were small then the rate calculation was subject to error. At day 42 the $2.5 \%$ error in phenol determination would give a $50 \%$ error in the $R 3$ rate. No methane was produced by these reactors.

\section{Conclusions}

The work leaves a number of questions open, but with some caution the following tentative conclusions on the startup of these anaerobic biofilm reactors can be given:

1) The degradation rates depended on concentrations in the reactor during the startup period, and generally follow Monod kinetics.

2) In spite of a more than two times higher biomass retention on porous glass carrier, the phenol degradation rates were essentially the same as with sand carrier.

3) Biomass development for phenol degradation on sand was only slightly promoted by low residence times.

4) Biomass development for molasses degradation appeared to be promoted more by high loading than by low residence times. Concentrations in the reactor seemed to be the fundamentally controlling factor, and residence times appeared to have little influence.

5) The reactors with short residence times (and high loading) exhibited higher specific biomass activities for both molasses and phenol.

6) The inoculum seemed to have the greatest influence of all variables on the molasses degradation startup. An active biofilm culture was much better than an activated sludge mixture. Starting a new reactor proved to be better than reinoculating the previously started reactor. This may have been due to the rather delicate balance required between acidogenic and methanogenic organisms, which was not quickly satisfied by simply reinoculating methanogens to an acidogenic biofilm.

\section{References}

1. Salkinoja-Salonen, M. S.; Nyns, E.-J.; Sutton, P. M.; Berg, L. v. d.; Wheatley, A. D.: Starting-up of an anaerobic fixed-film reactor. Wat. Sci. Tech. 15 (1983) 305-308

2. Heijnen, J. J.: Biological industrial waste-water treatment minimizing biomass production and maximizing biomass concentration. Ph.D. dissertation, Delft Technical University, Delft, The Netherlands (1984)

3. Shapiro, M.; Switzenbaum, M. S.: Initial anaerobic biofilm development. Biotech. Letters 6 (1984) 729-734

4. Bull, M. A.; Sterritt, R. M.; Lester, J. N.: An evaluation of four start-up regimes for anaerobic fluidized bed reactors. Biotech. Letters 5 (1984) 333-338

5. Gorriss, L. G. M.; Deursen van, J. M. A.; Drift, C. v. d.; Vogels, G. D.: Influence of waste water composition on biofilm development in laboratory methanogenic fluidized bed reactors. Appl Microbiol Biotechnol 29 (1988) 95-102

6. Gorriss, L. G. M.; Deursen van, J. M. A.; Drift, C. v. d.; Vogels, G. D.: Biofilm development in laboratory methanogenic fluidized bed reactors. Biotechnol Bioeng. 33 (1989) 687-693

Received August 29, 1989

I. J. Dunn

S. Petrozzi

Biological Reaction Engineering Group

Chemical Engineering Department

ETH

8092 Zurich

Switzerland 